\title{
On-Line Isotope Separation of Projectile Fragments Produced in Relativistic Heavy-Ion Reactions
}

\author{
Y. Nojiri, ${ }^{(a)}$ K. Matsuta, ${ }^{(a)}$ T. Minamisono, ${ }^{(a)} \mathrm{K}_{\text {. Sugimoto, }}^{(a)}$ \\ K. Takeyama, (a) H. Hamagaki, (b) S. Nagamiya, (b) K. Omala, (b) \\ Y. Shida, (b) I, Tanihata, (c) $T_{\text {T Kobayashi, (d) S. Matsuki, (e) }}$ \\ S. Shimoura, (e) J. R. Alonso, (i) G. Krebs, (i) T.J.M. Symons ${ }^{(1)}$
}
(a) Faculty of Science, Osaka University, Toyonaka 560, Japan
(b) INS, The University of Tokyo, Taneshi 188, Japan
(c) The Institute for Physical and Chemical Research, Wako 179, Japan
(d) National Laboratory for High Energy Physics, Ibaraki $30 j$, Japan
(e) Department of Physics, Faculty of Science, Kyoto University. Kyolo 606, Japan
(1) Lawrence Berkeley Laboralory, University of California, Berkeley, CA 94720, USA

\begin{abstract}
A new on-line separator was constructed and used successfully for the study on short-lived isotopes at the Bevalac at Lawrence Berkeley Laboratory. The isotopes were produced through projectile fragmentation processes of high energy heavy-ion reactions. Various isolopes were rigidity-separated by use of a beam line and, finally, the desired single isotope was range-analyzed to stop in a catcher. A large number of $\beta$-emitting ${ }^{21} \mathrm{~F}$ nuclei were successfully collecied and the nuclear lifetime was determined by detecting $\beta$ rays.
\end{abstract}

\section{Introduction}

Radioactive isotopes have played an important role in research, not only in nuclear structure but also in fundamental interactions, where the radioactive nucleus serves as a microlaboratory. In addition, they have been indispensable probes in investigating new solid state and electronic structures of rare impurities in metallic and non-metallic materials. Typically, $\beta$-radioactive nuclei have been used to inve, 
ture through nuclear moments, ${ }^{1}$ nuclear :eaction mechanisms, ${ }^{2}$ hyperfine interactions, ${ }^{3}$ and $\beta$ decays. ${ }^{3,4}$

In preparing $\beta$-radioactive nuclei for these investigations, on-line isotope separators connected directly with the primary beam line enable us to separate an isotope quickly. In the field of high energy heavy-ion nuclear physics it has been found that: 1) a wide variety of radioactive isotopes, including those far from the stability line, can be produced in large numbers through projectile fragmentation processes; 2) the nuclei of an isotope produced in these processes have surprisingly small momentum and angular spread after coming out of production targets. Those facts indicate that radioactive isotope beams (secondary beams) can be handled about as easily as primary beams used for conventional experiments.

At the Bevalac, the secondary beams of projectile fragments are produced through nuclear reactions between primary beams and targets at the exit of the Bevalac accelerator and are transferred through the existing beam lines originally prepared for primary beams. The secondary beams are analyzed by use of a series of dipole magnets and slit-jaws placed in certain positions in the beam lines. I single beam of radioactive isotopes desired for a particular experiment can be obtained at the end of the beam line, where the experiments are carried out.

In one successful experiment done with secondary beams, Tanihata et al. measured interaction cross sections of radioactive nuclei far from the stability line. ${ }^{5,6}$ In this experiment, light nuclei in the $\mathrm{s}$ and $\mathrm{p}$ shells, $\mathrm{He}$ isotopes, ${ }^{5} \mathrm{Li}$ isotopes, ${ }^{6}$ and $\mathrm{Be}$ isotopes ${ }^{6}$ were produced in projectile fragmentation processes between a Be target and a ${ }^{11} \mathrm{~B}$ or ${ }^{20} \mathrm{Ne}$ beam with energy of $\sim 800 \mathrm{MeV} /$ nucleon. After analysis and purification, these 
secondary beams were used to bombard three different targets, $\mathrm{Be}, \mathrm{C}$, and $\mathrm{Al}$, each between appproximately 5 and $20 \mathrm{~g} / \mathrm{cm}^{2}$ thick. In order to deduce interaction cross sections between the secondary beams and the targets, transmission rates of the secondary beams through these targets were observed.

In another application of secondiary beams to nuclear physics, it has been planned to determine magnetic moments of mirror pairs in f-shell nuclei ${ }^{7}$ by employing an NMR technique applicable to $\beta$-radioactive nuclei. ${ }^{1,3}$ In this technique, ions in the analyzed secondary beams are polarized with the tilted foil technique, ${ }^{8}$ and are stopped in a catcher material suitable for preserving their nuclear polarization for at least the duration of the nuclear lifetimes. At rest, well defined electromagnetic crystalline fields are available for the hyperfine interaction studies. For such heavy-ion implantations with the tilted foil technique, the kinetic energy of the ion has to be higher than 500 $\mathrm{keV} /$ nucleon, which is no problem for the secondary beam technique but poses considerable difficulty for the typical on-line isotope separator (ISOL) unless a post accelerator is employed.

\section{Experimental}

In the present experiment, $\beta$-decay rates of short-lived $\beta$-emitting ${ }^{21} \mathrm{~F}\left(I^{\pi}=5 / 2^{+}\right)$ were observed using a beam line (Beam 44) at the Bevalac, which is shown in Fig. 1. To produce ${ }^{21} \mathrm{~F}$ through the projectile fragmentation process a ${ }^{22} \mathrm{Ne}$ beam of $\sim 200$ $\mathrm{MeV} /$ nucleon was used to bombard a Be target $25.4 \mathrm{~mm}$ thick, which was set at the first focus (F1) of the extracted beam from the Bevalac. The expected production efficiency for radioactive isotopes is $0.1 \%$ of the incident beam. The net momentum 
spread for the isotope was calculated to be $|\Delta \mathrm{p} / \mathrm{p}|<0.5 \%$.

Four dipole magnets and quadrupole doublets were used to analyze and transfer the produced isotopes down to the experimental area (F5). At two momentum dispersive focus points of the beams (F2 and $F 4$ ), a desired isotope of a certain rigidity was separated by a pair of horizontal slit-jaws from other isotopes with different rigidities. The maximum rigidity was $65 \mathrm{kG} \cdot \mathrm{m}$, and the momentum acceptance was $\pm 0.65 \%$. Momentum dispersion of the beams at F2 is designed to be $\Delta x /(\Delta p / p)=25 \mathrm{~mm} / \%$. In between $F 2$ and F4, the beams have achromatic focus at F3, where two pairs of slitjaws were prepared as cleaning slits to reject scattered particles with different trajectories from the main beams. The total length from the target to the catcher was $\mathbf{5 5} \mathrm{m}$.

In order to stop a desired isotope in the thin catcher, a plastic plate $2 \mathrm{~mm}$ thick, a set of variable energy wedge absorbers made of thick plastic was employed in the beam course to control the energy of the isotope before it was caught. To determine the half life of ${ }^{21} \mathrm{~F}$ nuclei, time spectra of $\boldsymbol{\beta}$ rays from those nuclei stopped in the catcher were measured. For those measurements two sets of plastic scintillator counter telescopes were placed above and below the stopper. Two thin $\Delta \mathrm{E}$-ccunters (1 $\mathrm{mm}$ thick each) and one thick E-counter ( $30 \mathrm{~mm}$ thick) were used in each telescope in order to reduce detection of background radiations. The E-counter was thick enough to stop the $\beta$ rays from ${ }^{21} \mathrm{~F}$ decay (maximum $\beta$-ray energy is $5.7 \mathrm{MeV}$ ). The beam pulse gating method was used to separate counting periods of $\beta$ rays from production periods of the nuclei. For the case of ${ }^{21} \mathrm{~F}, 3$ ordinary beam spills ( $4 \mathrm{sec}$ apart) of the Bevalac were used for production, followed by a $45-\mathrm{sec}$ period of counting $\beta$ rays. Beam pulse gating was controlled through gating the if oscillator of the Bevatron. The half life of ${ }^{21} F$ was 
measured by multi-scaling the pulses for the $\beta$ detectors. The initial counting rate of the $\beta$ particles was as many as $2000 \mathrm{c} / \mathrm{sec}$.

\section{Results and Discussions}

The present result of ${ }^{2 \mathrm{i}} \mathrm{F}$ decay is shown in Fig. 2. The time spectrum was fitted to a decay function consisting of two exponential decays and a constant background. The minority group among the decaying components was identified as ${ }^{20} \mathrm{~F}$, which was produced through nuclear reactions between the separated isotope beams and the energy absorber placed in front of the stopper. The decay constant for ${ }^{20} \mathrm{~F}$ in the spectrum was chosen in the analyses to be $T_{1 / 2}=(11.03 \pm 0.06)$ sec, also shown in Fig. 2, which was measured in the next lifetime measurement on ${ }^{20} \mathrm{~F}$ and was consistent with known data.

The deduced half life of ${ }^{21} \mathrm{~F}$ was $(4.21 \pm 0.03)$ sec. The previous values reported for ${ }^{21}$ half life were $(4.21 \pm 0.05) \mathrm{sec}^{9}{ }^{9}(4.34 \pm 0.04) \mathrm{sec},{ }^{10}$ and $(4.158 \pm 0.020)$ sec. $^{11}$ These values are consistent within the errors, except for the one in Ref. 10 in which the uncertainties for the results were considered to be underestimated as discussed in Ref. 11.

In summary the present on-line separator has been successfully used for determination of lifetime of a $\beta$-radioactive nucleus. In the near future, the apparatus will be used for magnetic moment measurements of $\beta$-radioactive nuclei in the f-shell. ${ }^{7}$ For these experiments, an NMR technique applicable to $\beta$-radioactive nuclei will be combined with the separator. This will surely open up new applications of the technique to much wider varieties of studies using $\beta$-radioactive nuclei produced through relativistic heavy-ion reactions. 


\section{Acknowledgments}

The present work was supported in part by the Yamada Science Foundation and the program for INS-LBL ' llaborative Research and by a Grant-in-Aid for Scientific Research from the Ministry of Education, Science, and Culture of Japan. Also the study is partially supported by U.S. DOE under contract No. DE-AC03-76SF00098, and by the Japan-U.S. Cooperative Research Program of the Japan Society for the Promotion of Science.

\section{References}

1. K. Nakai, Hyp. Int. 21 (1985), 1.

2. K. Sugimoto et al., Phys. Rev. Lett. 39 (1877), 323.

3. T. Minamisono, Hyp. Int. 21 (1985), 103.

4. Y. Masuda et al., Phys. Rev. Lett. 43 (1979), 1083.

5. I. Tanihata et al., Phys. Lett. $160 \mathrm{~B}$ (1985), 380.

6. I. Tanihata et al., Phys. Rev. Lett. 55 (1985), 2676.

7. T. Minamisono et al., Bevalac Experiment E732H (1983).

8. H. G. Berry et al., Ann. Rev. Nucl. Par. Sci. 32 (1982), 1.

Y. Nojiri et al., Phys. Rev. Lett. 51 (1983), 180.

9. M. E. Bunker et al., Bull. Am. Phys. Soc. 8 (1063), 317.

10. J.L.C. Ford et al., Nucl. Phys. 63 (1965), 588.

11. E. K. Warburton et al., Phys. Rev. C23 (1981), 1234. 


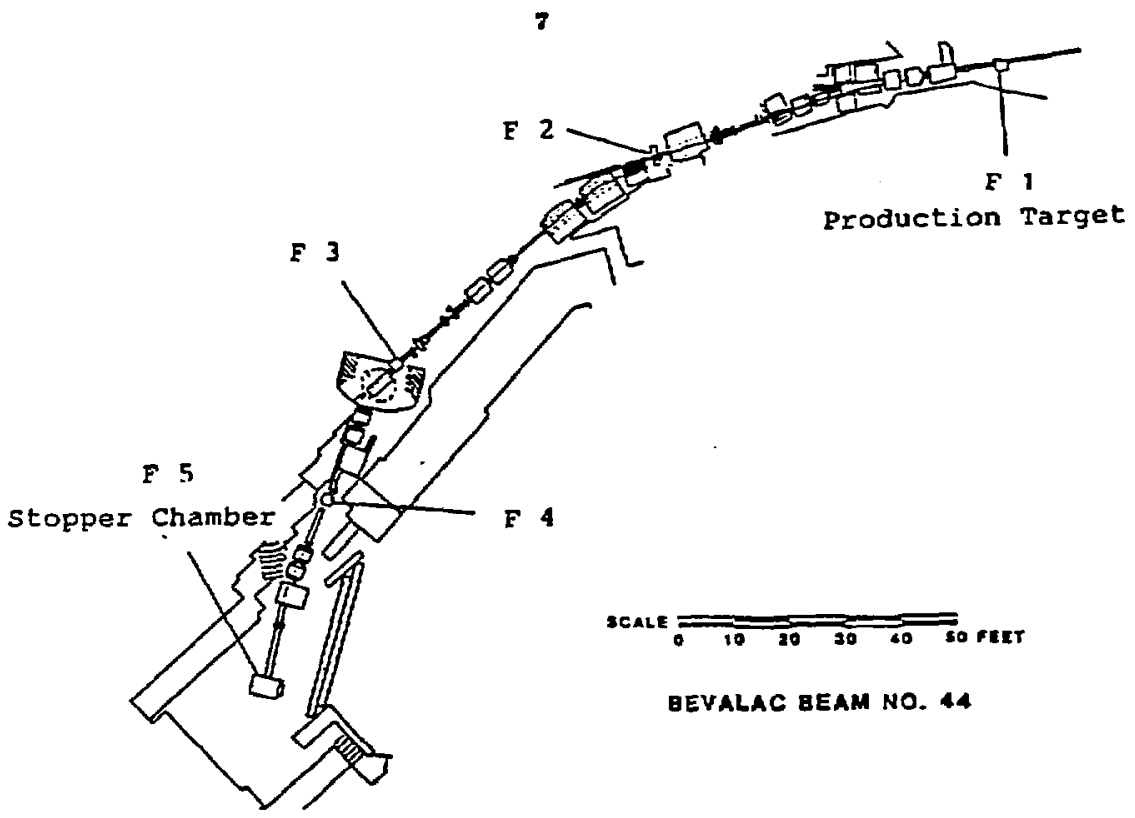

Fig. 1. Beam transport system in Beam 44 at the Bevalac.
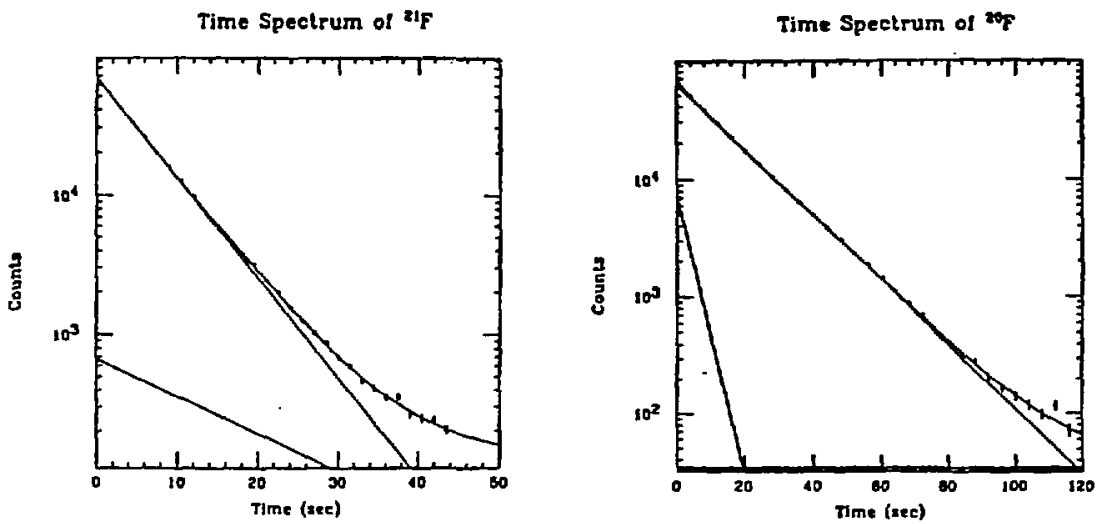

Fig. 2. Typical $\beta$-ray time spectra of ${ }^{21} \mathrm{~F}$ and ${ }^{20} \mathrm{~F}$. The theoretical lines best fitted to the data consist of 3 components that are also separately shown, respectively. 
This report was done with aupport from the Department of Enerty. Any conclusions or opinion expressed in this report represent solely thowe of the author(s) and not necesurily those of The Rezents of the University of Californit, the Lawrence Berkeley Laboratory or the Defartment of Energy.

Reference to a company or product name does not imply approval or recommendation of the product by the University of Californin or the U.S. Department of Energy to the exclusion of others that may be suitable. 